\title{
An Optimal Bivariate Polynomial Interpolation Basis for the Application of the Evaluation-Interpolation Technique
}

\author{
Dimitris Varsamis $^{1, *}$, Nicholas Karampetakis ${ }^{2}$ and Paris Mastorocostas ${ }^{1}$ \\ ${ }^{1}$ Department of Informatics \& Communications, Technological Educational Institute of Serres, 62124 Serres, Greece \\ ${ }^{2}$ Department of Mathematics, Aristotle University of Thessaloniki, 54124 Thessaloniki, Greece
}

Received: 31 May. 2013, Revised: 14 Sep. 2013, Accepted: 15 Sep. 2013

Published online: 1 Jan. 2014

\begin{abstract}
A new basis of interpolation points for the special case of the Newton two variable polynomial interpolation problem is proposed. This basis is implemented when the upper bound of the total degree and the degree in each variable is known. It is shown that this new basis under certain conditions (that depends on the degrees of the interpolation polynomial), coincides either with the known triangular/rectangular basis or it is a polygonal basis. In all cases it uses the least interpolation points with further consequences to the complexity of the algorithms that we use.
\end{abstract}

Keywords: bivariate polynomial, Newton interpolation, determinant, interpolation points

\section{Introduction}

The problem of the approximation of a complicated function $f$ by a simple function $g$ is called interpolation. A few distinct known data points from the original function can be used to create an interpolation based on a simpler function. When the function $g$ is a polynomial we call the method polynomial interpolation. The bivariate polynomial interpolation is today a basic subject of Approximation Theory and Numerical Analysis [1,2] with applications to many mathematical problems $[3,4]$. The problem of multivariate interpolation has been the concern of many scientists $[5,6,7,8,9]$. Some of the most known multivariate interpolation methods are: a) the use of a multivariate Vandermode matrix and its LU factorization [9], b) the Lagrange interpolation [10,11] c) the Hermite-Birkhoff interpolation [12] and d) the Newton-form interpolation [8,10,13].

An interpolation problem is defined to be poised if it has a unique solution. The two-variable interpolation problem is not always poised. It is poised when we use the known triangular/rectangular basis of interpolation points [10]. In particular, the two-variable Newton interpolation can be implemented in two bases of interpolation points [13] : triangular and rectangular as we see below.
The interpolating polynomial with the form

$$
p(x, y)=\sum_{i=0}^{n} \sum_{j=0}^{n-i} a_{i, j} x^{i} y^{j}
$$

has total degree $n=\operatorname{deg}[p(x, y)]$ and defined uniquely in the following set of $N=\left(\begin{array}{c}n+2 \\ n\end{array}\right)$ interpolation points (triangular basis)

$$
S_{\Delta}^{(n)}=\left\{\left(x_{i}, y_{j}\right) \mid i, j \in \mathbb{N}, i+j \leq n\right\}
$$

(1) can be rewritten as follows:

$$
p(x, y)=X^{T} \cdot P \cdot Y
$$

where

$$
P=\left(\begin{array}{cccccc}
p_{0,0}^{(0)} & p_{0,1}^{(1)} & p_{0,2}^{(2)} & \cdots & p_{0, n-1}^{(n-1)} & p_{0, n}^{(n)} \\
p_{1,0}^{(1)} & p_{1,1}^{(1)} & p_{1,2}^{(2)} & \cdots & p_{1, n-1}^{(n-1)} & 0 \\
p_{2,0}^{(2)} & p_{2,1}^{(2)} & p_{2,2}^{(2)} & \cdots & 0 & 0 \\
\vdots & \vdots & \vdots & \ddots & \vdots & \vdots \\
p_{n-1,0}^{(n-1)} & p_{n-1,1}^{(n-1)} & 0 & \cdots & 0 & 0 \\
p_{n, 0}^{(n)} & 0 & 0 & \cdots & 0 & 0
\end{array}\right)
$$

\footnotetext{
* Corresponding author e-mail: dvarsam @ teiser.gr
} 
and

$$
\begin{aligned}
& X=\left(\begin{array}{c}
1 \\
x-x_{0} \\
\left(x-x_{0}\right)\left(x-x_{1}\right) \\
\vdots \\
\left(x-x_{0}\right)\left(x-x_{1}\right) \cdots\left(x-x_{n-1}\right)
\end{array}\right) \\
& 1 \\
& Y=\left(\begin{array}{c}
y-y_{0} \\
\left(y-y_{0}\right)\left(y-y_{1}\right) \\
\vdots \\
\left(y-y_{0}\right)\left(y-y_{1}\right) \cdots\left(y-y_{n-1}\right)
\end{array}\right)
\end{aligned}
$$

with $P \in \mathbb{R}^{(n+1) \times(n+1)}, X \in \mathbb{R}[x]^{(n+1)}$ and $Y \in \mathbb{R}[y]^{(n+1)}$. Additionally, the interpolating polynomial with the form

$$
p(x, y)=\sum_{i=0}^{k_{1}} \sum_{j=0}^{k_{2}} a_{i, j} x^{i} y^{j}
$$

has total degree in terms of $x$ (resp. in terms of $y$ ) $k_{1}=$ $\operatorname{deg}_{x}[p(x, y)]$ (resp. $\left.k_{2} \operatorname{deg}_{y}[p(x, y)]\right)$ and defined uniquely in the following set of $\tilde{N}=\left(k_{1}+1\right)\left(k_{2}+1\right)$ interpolation points (rectangular basis)

$$
\tilde{S}_{\Delta}^{\left(k_{1}, k_{2}\right)}=\left\{\left(x_{i}, y_{j}\right) \mid i, j \in \mathbb{N}, i \leq k_{1}, j \leq k_{2}\right\}
$$

(3) can be rewritten as

$$
p(x, y)=\tilde{X}^{T} \cdot \tilde{P} \cdot \tilde{Y}
$$

where (in case $k_{1}>k_{2}$ )

$$
\tilde{P}=\left(\begin{array}{ccccc}
p_{0,0}^{(0)} & p_{0,1}^{(1)} & p_{0,2}^{(2)} & \cdots & p_{0, k_{2}}^{\left(k_{2}\right)} \\
p_{1,0}^{(1)} & p_{1,1}^{(1)} & p_{1,2}^{(2)} & \cdots & p_{1, k_{2}}^{\left(k_{2}\right)} \\
p_{2,0}^{(2)} & p_{2,1}^{(2)} & p_{2,2}^{(2)} & \cdots & p_{2, k_{2}}^{\left.k_{2}\right)} \\
\vdots & \vdots & \vdots & \ddots & \vdots \\
p_{k_{2}, 0}^{\left(k_{2}\right)} & p_{k_{2}, 1}^{\left(k_{2}\right)} & p_{k_{2}, 2}^{\left(k_{2}\right)} & \cdots & p_{k_{2}, k_{2}}^{\left(k_{2}\right)} \\
\vdots & \vdots & \vdots & \ddots & \vdots \\
p_{k_{1}, 0}^{\left(k_{1}\right)} & p_{k_{1}, 1}^{\left(k_{1}\right)} & p_{k_{1}, 2}^{\left(k_{1}\right)} & \cdots & p_{k_{1}, k_{2}}^{\left(k_{1}\right)}
\end{array}\right)
$$

and

$$
\begin{gathered}
\tilde{X}=\left(\begin{array}{c}
1 \\
x-x_{0} \\
\left(x-x_{0}\right)\left(x-x_{1}\right) \\
\vdots \\
\left(x-x_{0}\right)\left(x-x_{1}\right) \cdots\left(x-x_{k_{1}-1}\right) \\
1 \\
y-y_{0} \\
\vdots \\
\left(y-y_{0}\right)\left(y-y_{1}\right) \\
\vdots \\
\left(y-y_{0}\right)\left(y-y_{1}\right) \cdots\left(y-y_{k_{2}-1}\right)
\end{array}\right)
\end{gathered}
$$

with $\tilde{P} \in \mathbb{R}^{\left(k_{1}+1\right) \times\left(k_{2}+1\right)}, \tilde{X} \in \mathbb{R}[x]^{\left(k_{1}+1\right)}$ and $\tilde{Y} \in \mathbb{R}[y]^{\left(k_{2}+1\right)}$ where $p_{i, j}^{(k)}$ in $P$ and $\tilde{P}$ are known as divided differences and are computed in [13] by using the following recursive formula

$$
p_{i, j}^{(k)}:=\left\{\begin{array}{cc}
\frac{p_{i, j}^{(k-1)}-p_{i-1, j}^{(k-1)}}{x_{i}-x_{i-k}} & \text { if }(j<k \wedge i \geq k) \\
\frac{p_{i, j}^{(k-1)}-p_{i, j-1}^{(k-1)}}{y_{j}-y_{j-k}} & \text { if }(i<k \wedge j \geq k) \\
\frac{p_{i, j}^{(k-1)}+p_{i-1, j-1}^{(k-1)}-p_{i-1, j}^{(k-1)}-p_{i, j-1}^{(k-1)}}{\left(x_{i}-x_{i-k}\right)\left(y_{j}-y_{j-k}\right)} & \text { if }(i \geq k \wedge j \geq k) \\
p_{i, j}^{(k-1)} & \text { if }(i<k \wedge j<k)
\end{array}\right.
$$

There are many problems in applied mathematics where the function $f$ that we need to interpolate is already a two-variable polynomial or a two-variable polynomial matrix with known upper bounds both of the total degree and the degree for each variable. For the computation of the interpolating polynomial of $f$ we use the technique "evaluation-interpolation". That means, in the first step we calculate the values of $f$ on a set of interpolation points $\left(x_{i}, y_{j}\right)$ whereas in the second step we compute the interpolating polynomial with interpolation techniques. Such kind of problems are the following : the computation of the determinant of a two-variable polynomial matrix [13], the computation of the greatest common divisor of two-variable polynomials [14] the computation of the inverse matrix of a two variable polynomial matrix [15], [16], [13] etc.. To make that clear we give the following example.

Example 1.Let the two-variable polynomial matrix

$A(x, y)=\left[a_{i, j}(x, y)\right]=\left[\begin{array}{cccc}x^{4}+y^{2} & 2 & 3 & x^{3}-x y^{5} \\ x^{9}-y^{2} & x+y & x^{3} y^{2} & x^{2}+y^{9} \\ y & x^{3}+x y^{2} & x & x^{4} y^{6}+y^{8} \\ 1 & x^{2}-y^{3} & y & x^{3} y^{3}\end{array}\right]$

We know that an upper bound of the total degree of the polynomial $p(x, y)=\operatorname{det}(A(x, y))$ is given by the sum of the greatest total degrees in each column or row of all polynomials in matrix $A(x, y)$ i.e.

$$
\begin{aligned}
n=\min & \left\{\sum_{i=1}^{4}\left(\max _{1 \leq j \leq 4}\left\{\operatorname{deg}\left[a_{i, j}(x, y)\right]\right\}\right),\right. \\
& \left.\sum_{j=1}^{4}\left(\max _{1 \leq i \leq 4}\left\{\operatorname{deg}\left[a_{i, j}(x, y)\right]\right\}\right)\right\} \\
= & \min \{6+9+10+6,9+3+5+10\}=27
\end{aligned}
$$


Similarly, an upper bound of the degree $k_{1}$ (resp. $k_{2}$ ) in terms of $x$ (resp. $y$ ) of $p(x, y)$ is given by

$$
\begin{aligned}
k_{1}=\min & \left\{\sum_{i=1}^{4}\left(\max _{1 \leq j \leq 4}\left\{\operatorname{deg}_{x}\left[a_{i, j}(x, y)\right]\right\}\right),\right. \\
& \left.\sum_{j=1}^{4}\left(\max _{1 \leq i \leq 4}\left\{\operatorname{deg}_{x}\left[a_{i, j}(x, y)\right]\right\}\right)\right\} \\
& =\min \{4+9+4+3,9+3+3+4\}=19
\end{aligned}
$$

and

$$
\begin{aligned}
k_{2}=\min & \left\{\sum_{i=1}^{4}\left(\max _{1 \leq j \leq 4}\left\{\operatorname{deg}_{y}\left[a_{i, j}(x, y)\right]\right\}\right)\right. \\
& \left.\sum_{j=1}^{4}\left(\max _{1 \leq i \leq 4}\left\{\operatorname{deg}_{y}\left[a_{i, j}(x, y)\right]\right\}\right)\right\} \\
& =\min \{5+9+8+3,2+3+2+9\}=16
\end{aligned}
$$

Therefore, either we find an interpolating polynomial with total degree $n=27$ by using $N=\left(\begin{array}{c}n+2 \\ n\end{array}\right)=406$ interpolation points on a triangular basis or we find an interpolating polynomial with total degree $k_{1}=19$ (resp. $k_{2}=16$ ) in terms of $x$ (resp. y) by using $\tilde{N}=\left(k_{1}+1\right)\left(k_{2}+1\right)=340$ interpolation points on a rectangular basis. Note that $n<k_{1}+k_{2}<2 \cdot n$.

In order to compute the determinant of the polynomial matrix in the above example, we need to compute the determinants of $A(x, y)$ at the interpolation points $\left(x_{i}, y_{i}\right)$ of either a triangular or a rectangular basis. The less interpolation points that we get, the less computational cost will have the solution to our problem. Therefore, certain questions still remaining as concerns the set of the interpolation points that we shall use. In the above example, it is easily seen that by selecting the rectangular basis we shall have the least computational cost due to less interpolation points (340 instead of 406 for the triangular basis). Is there any other basis with less interpolation points for the above example and the specific upper bound conditions given? As we propose in Section 2, in that kind of problems we can always select a new polygonal basis, which in certain cases coincides with the ones of triangular/rectangular basis and in the most of the cases use less interpolation points from both of them. Furthermore, in Section 3 we present a formula that help us to select the appropriate basis according to the known upper bounds in the total degree and the degree of each variable of the interpolating polynomial. The whole theory is illustrated by specific examples.

\section{Bivariate interpolation on a polygonal basis}

The aim of this section, is to propose a new polygonal basis, for the Newton interpolation of a two-variable polynomial with specific upper bound on the total degree and the degree in terms of each variable. As we shall see, the proposed basis will use less interpolation points than the ones of triangular/rectangular basis. We start by a simple example, in order to make clear why all the interpolation points in triangular/rectangular basis are not needed for the evaluation of the coefficients of the interpolating polynomial.

Example 2.Let the function

$$
f(x, y)=3 x^{2}+2 x^{2} y+x y^{2}-y^{2}
$$

Suppose that the only things we know is that $f(x, y)$ is a polynomial with:

-upper bound of the total degree $n=3$,

-and upper bound of degree for the variable $x$ (resp. $y$ ), $k_{1}=2$ (resp. $\left.k_{2}=2\right)$

and we know its values at any interpolation points $\left(x_{i}, y_{i}\right)$. Triangular basis

Let the set of $N=\left(\begin{array}{c}n+2 \\ n\end{array}\right)=10$ interpolating points of the triangular basis

$S_{\Delta}^{(3)}=\left\{\left(x_{i}=i, y_{j}=j\right) \mid i=0,1,2,3, j=0,1,2,3, i+j \leq 3\right\}$

and the initial values $p_{i, j}^{(0)}=f\left(x_{i}, y_{j}\right)$ given by the following table

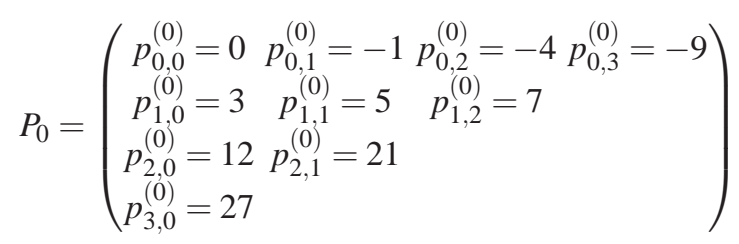

For $\mathbf{k}=\mathbf{1}(\mathbf{1}) \mathbf{n}$, compute the tables of order $k$ by using the recursive formula (5) [13].

The Newton interpolating polynomial is the following:

$$
p(x, y)=X^{T} \cdot P_{3} \cdot Y=3 x^{2}+2 x^{2} y+x y^{2}-y^{2}
$$

where

$$
X=\left(\begin{array}{c}
1 \\
x \\
x(x-1) \\
x(x-1)(x-2)
\end{array}\right), \quad Y=\left(\begin{array}{c}
1 \\
y \\
y(y-1) \\
y(y-1)(y-2)
\end{array}\right)
$$

and

$$
P_{3}=\left(\begin{array}{lll}
p_{0,0}^{(0)}=0 & p_{0,1}^{(1)}=-1 & p_{0,2}^{(2)}=-1 \mathbf{p}_{\mathbf{0}, \mathbf{3}}^{(\mathbf{3})}=\mathbf{0} \\
p_{1,0}^{(1)}=3 & p_{1,1}^{(1)}=3 & p_{1,2}^{(2)}=1 \\
p_{2,0}^{(2)}=3 & p_{2,1}^{(2)}=2 \\
\mathbf{p}_{\mathbf{3}, \mathbf{0}}^{(\mathbf{3})}=\mathbf{0} &
\end{array}\right)
$$

\section{Rectangular basis}

Let the set of $\tilde{N}=\left(k_{1}+1\right)\left(k_{2}+1\right)=9$ interpolating points of the rectangular basis

$$
\tilde{S}_{\Delta}^{(2,2)}=\left\{\left(x_{i}=i, y_{j}=j\right) \mid i=0,1,2, j=0,1,2\right\}
$$


and the initial values $p_{i, j}^{(0)}=f\left(x_{i}, y_{j}\right)$ given by the following table

$$
\tilde{P}_{0}=\left(\begin{array}{ccc}
p_{0,0}^{(0)}=0 & p_{0,1}^{(0)}=-1 & p_{0,2}^{(0)}=-4 \\
p_{1,0}^{(0)}=3 & p_{1,1}^{(0)}=5 & p_{1,2}^{(0)}=7 \\
p_{2,0}^{(0)}=12 & p_{2,1}^{(0)}=21 & p_{2,2}^{(0)}=32
\end{array}\right)
$$

For $\mathbf{k}=\mathbf{1}(\mathbf{1}) \tilde{\mathbf{n}}$, where $\tilde{n}=\max \left\{k_{1}, k_{2}\right\}=\max \{2,2\}=2$, compute the tables of order $k$ by using the recursive formula (5) [13].

The Newton interpolating polynomial is the following:

$$
p(x, y)=\tilde{X}^{T} \cdot \tilde{P}_{2} \cdot \tilde{Y}=3 x^{2}+2 x^{2} y+x y^{2}-y^{2}
$$

where

$$
\tilde{X}=\left(\begin{array}{c}
1 \\
x \\
x(x-1)
\end{array}\right), \quad \tilde{Y}=\left(\begin{array}{c}
1 \\
y \\
y(y-1)
\end{array}\right)
$$

and

$$
\tilde{P}_{2}=\left(\begin{array}{ccc}
p_{0,0}^{(0)}=0 & p_{0,1}^{(1)}=-1 & p_{0,2}^{(2)}=-1 \\
p_{1,0}^{(1)}=3 & p_{1,1}^{(1)}=3 & p_{1,2}^{(2)}=1 \\
p_{2,0}^{(2)}=3 & p_{2,1}^{(2)}=2 & \mathbf{p}_{\mathbf{2}, 2}^{(2)}=\mathbf{0}
\end{array}\right)
$$

From the above example we observe that :

-the elements $p_{3,0}^{(3)}$ and $p_{0,3}^{(3)}$ when we use the triangular basis are equal to zero because we do not have the terms $x^{3}$ or $y^{3}$. Similarly, the element $p_{2,2}^{(2)}$ when we use the rectangular basis is equal to zero because we do not have the term $x^{2} y^{2}$.

-the interpolating polynomial can be calculated either by using the set $S_{\Delta}^{(n)}$ (triangular basis) or by using the set $\tilde{S}_{\Delta}^{\left(k_{1}, k_{2}\right)}$ (rectangular basis). However, although the sets $S_{\Delta}^{(n)}$ and $\tilde{S}_{\Delta}^{\left(k_{1}, k_{2}\right)}$ have common interpolation points, the one is not a subset of the other.

The aim of the following lemma is to prove our first observation in the previous example.

Lemma 1.Let a Newton interpolating polynomial $p(x, y)$ with total degree $n$. Then, the divided difference $p_{m, k}^{(\max \{m, k\})}$ defined in (5) with $m+k>n$ is equal to zero.

Proof. Note that $p_{m, k}^{(\max \{m, k\})}$ with $m+k>n$, is the coefficient of the term

$$
p_{m, k}^{(\max \{m, k\})} \prod_{i=0}^{m-1}\left(x-x_{i}\right) \prod_{i=0}^{k-1}\left(y-y_{i}\right)
$$

in (4) and thus creates the power $x^{m} \cdot y^{k}$ of total degree $m+k>n$. Since $p(x, y)$ has total degree $n$ we have that $p_{m, k}^{(\max \{m, k\})}=0$ which verifies the lemma.
This is the reason why $\mathbf{p}_{\mathbf{2}, \mathbf{2}}^{(\mathbf{2})}$ is equal to zero in the above example when we use the rectangular basis . Similarly, someone can show the following lemma.

Lemma 2.Let a Newton interpolating polynomial $p(x, y)$ with degree $k_{1}$ (resp. $k_{2}$ ) in terms of $x$ (resp. $y$ ). Then, the divided difference $p_{k, n-k}^{(n)}$ with $k=0,1, \ldots, n$ defined in (5) with either $k>k_{1}$ or $n-k>k_{2}$ is equal to zero.

This is the reason why $\mathbf{p}_{\mathbf{3}, \mathbf{0}}^{(\mathbf{3})}$ and $\mathbf{p}_{\mathbf{0}, \mathbf{3}}^{(\mathbf{3})}$ are becoming zero when we use the triangular basis.

Theorem 1.The set of interpolation points given by the polygonal basis

$$
\begin{gathered}
\hat{S}_{\Delta}^{\left(n, k_{1}, k_{2}\right)}=S_{\Delta}^{(n)} \bigcap \tilde{S}_{\Delta}^{\left(k_{1}, k_{2}\right)}= \\
=\left\{\left(x_{i}, y_{j}\right) \mid i=0,1, \ldots, k_{1}, j=0,1, \ldots, k_{2}, i+j \leq n\right\}
\end{gathered}
$$

are enough in order to evaluate uniquely the coefficients of a Newton interpolating polynomial $p(x, y)$ with total degree $n$, and total degree $k_{1}$ (resp. $k_{2}$ ) in terms of $x$ (resp. $y)$.

Proof.According to Lemma 1 the final divided differences $p_{i, j}^{(\max \{i, j\})}$ with $i+j>n$ are equal to zero since the total degree of $p(x, y)$ is equal to $n$. As we can see from (5) the computation of $p_{i, j}^{(\max \{i, j\})}$ is based on the values of $p_{l, m}^{(k)}$ with $k<\max \{i, j\}, l \leq i$ and $m \leq j$. Although the values of $p_{i, j}^{(k)}$ for $k<\max \{i, j\}$ may not be zero, their computation are not necessary since they have impact only on the evaluation of divided differences of the form $p_{l, m}^{(\max \{i, j\})}$ with $l \geq i$ and $m \geq j$ which gives rise to zero coefficients of the Newton interpolating polynomial since $l+m \geq i+j>n$.

According to Lemma 2 the divided difference $p_{i, n-i}^{(n)}$ with $i=0,1, \ldots, n$ defined in (5) with either $i>k_{1}$ or $n-i>$ $k_{2}$ is equal to zero. The computation of $p_{i, n-i}^{(n)}$ with $i>k_{1}$ and $n-i>k_{2}$ is based on the values of $p_{l, m}^{(k)}$ with $k \leq n, l \leq i$ and $m \leq n-i$. Although the values of $p_{i, n-i}^{(k)}$ with $k<n$ may not be zero, their computation are not necessary since they have impact only on the evaluation of divided differences of the form $p_{l, m}^{(k)}$ with $l \geq i$ and $m \geq n-i$ which gives rise to zero coefficients of the Newton interpolating polynomial since $l \geq i>k_{1}$ and $m \geq n-i>k_{2}$.

From the above we conclude, that the Newton interpolating polynomial $p(x, y)$ can be computed by using only the initial values $p\left(x_{i}, y_{j}\right)=p_{i, j}^{(0)}$ where $i \leq k_{1}$, $j \leq k_{2}$ and $i+j \leq n$ which verifies the Theorem.

Due to (6) the number of interpolation points that we need for the polygonal basis $\hat{S}_{\Delta}^{\left(n, k_{1}, k_{2}\right)}$ is $\hat{N}=\left(\begin{array}{c}n+2 \\ n\end{array}\right)-\left(S_{n-k_{1}}+S_{n-k_{2}}\right)$. The recursive formula for the calculation of divided differences (5) and the 
algorithm for the calculation of the Newton interpolating polynomial given in [13] can now be used in this polygonal basis as we can see in the following Example.

Example 3.Consider the Example 2. Denote the set of

$\hat{N}=\left(\begin{array}{c}n+2 \\ n\end{array}\right)-\left(S_{n-k_{1}}+S_{n-k_{2}}\right)=\left(\begin{array}{c}3+2 \\ 3\end{array}\right)-\left(S_{1}+S_{1}\right)=8$ interpolating points given by the set (polygonal basis)

$\hat{S}_{\Delta}^{(3,2,2)}=\left\{\left(x_{i}=i, y_{j}=j\right) \mid i=0,1,2, j=0,1,2, i+j \leq 3\right\}$

and the values of the function $f(x, y)$ on that points as given by the table

$$
\begin{array}{c|ccc}
f\left(x_{i}, y_{j}\right) & y_{0}=0 & y_{1}=1 & y_{2}=2 \\
\hline x_{0}=0 & 0 & -1 & -9 \\
x_{1}=1 & 3 & 5 & 7 \\
x_{2}=2 & 12 & 21 &
\end{array}
$$

Step 1: Construction of the table with initial values

$$
\hat{P}_{0}=\left(\begin{array}{ccc}
p_{0,0}^{(0)}=0 & p_{0,1}^{(0)}=-1 & p_{0,2}^{(0)}=-4 \\
p_{1,0}^{(0)}=3 & p_{1,1}^{(0)}=5 & p_{1,2}^{(0)}=7 \\
p_{2,0}^{(0)}=12 & p_{2,1}^{(0)}=21 &
\end{array}\right)
$$

Step 2: For $\quad \mathbf{k}=\mathbf{1}(\mathbf{1}) \tilde{\mathbf{n}}, \quad$ where $\tilde{n}=\max \left\{k_{1}, k_{2}\right\}=\max \{2,2\}=2$, compute the tables of order $k$, where $p_{i, j}^{(k)}$ are given by the recursive formula 5 . For $\mathbf{k}=\mathbf{1}$, the first order table of divided differences is given by

$$
\hat{P}_{1}=\left(\begin{array}{lll}
p_{0,0}^{(0)}=0 & p_{0,1}^{(1)}=-1 & p_{0,2}^{(1)}=-3 \\
p_{1,0}^{(1)}=3 & p_{1,1}^{(1)}=3 & p_{1,2}^{(1)}=5 \\
p_{2,0}^{(1)}=9 & p_{2,1}^{(1)}=7 &
\end{array}\right)
$$

For $\mathbf{k}=\mathbf{2}$, the second order table of divided differences is given by

$$
\hat{P}_{2}=\left(\begin{array}{lll}
p_{0,0}^{(0)}=0 & p_{0,1}^{(1)}=-1 & p_{0,2}^{(2)}=-1 \\
p_{1,0}^{(1)}=3 & p_{1,1}^{(1)}=3 & p_{1,2}^{(2)}=1 \\
p_{2,0}^{(2)}=3 & p_{2,1}^{(2)}=2 &
\end{array}\right)
$$

Step 3: The Newton interpolating polynomial in polygonal basis is the following:

$$
p(x, y)=\hat{X}^{T} \cdot \hat{P} \cdot \hat{Y}=x^{2}+x^{2} y+y^{2}
$$

where $\hat{P}=\hat{P}_{2}$ with zero at the position $(3,3)$ and

$$
\hat{X}=\left(\begin{array}{c}
1 \\
x \\
x(x-1)
\end{array}\right), \quad \hat{Y}=\left(\begin{array}{c}
1 \\
y \\
y(y-1)
\end{array}\right)
$$

which coincides with the results given in Example 2

The main advantage of polygonal basis is that it uses less interpolation points when $n<k_{1}+k_{2}<2 \cdot n$ (see Table 1 and Figure 1).
Table 1: The number of interpolation points in all bases when $n$ is constant (10) and $k_{1}$ (resp. $k_{2}$ ) gets values from 0 to 10

\begin{tabular}{llll}
\hline$\left(n, k_{1}, k_{2}\right)$ & $N$ & $\tilde{N}$ & $\hat{N}$ \\
\hline$(10,2,8)$ & 66 & 27 & 27 \\
$(10,3,8)$ & 66 & 36 & 35 \\
$(10,4,9)$ & 66 & 50 & 44 \\
$(10,5,9)$ & 66 & 60 & 50 \\
$(10,6,9)$ & 66 & 70 & 55 \\
$(10,7,9)$ & 66 & 80 & 59 \\
$(10,8,9)$ & 66 & 90 & 62 \\
$(10,9,9)$ & 66 & 100 & 64 \\
$(10,10,9)$ & 66 & 110 & 65 \\
$(10,10,10)$ & 66 & 121 & 66 \\
\hline
\end{tabular}

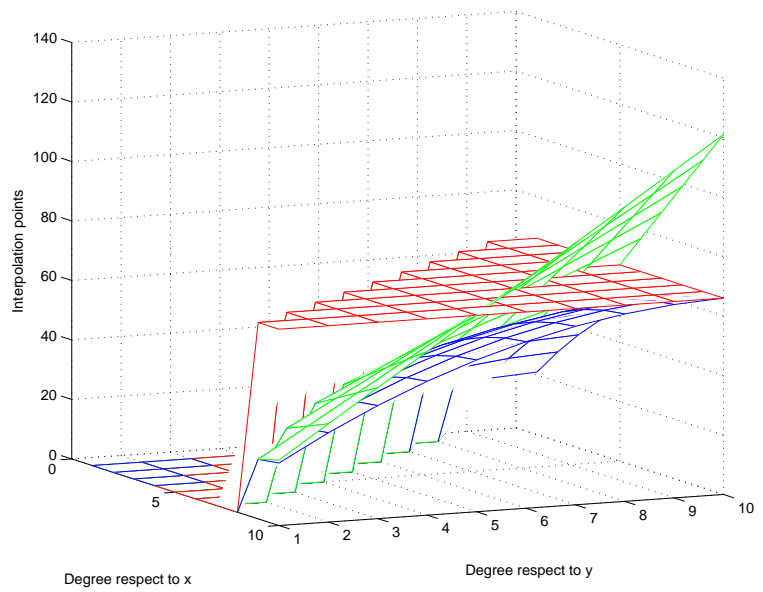

Fig. 1: The number of interpolation points in all bases (red: triangular, green: rectangular and blue: polygonal) when $n$ is constant (10) and $k_{1}$ (resp. $k_{2}$ ) gets values from 0 to 10

\section{An optimal basis for the evaluation of the Newton two-variable interpolating polynomial}

We consider as "optimal basis" the set with the least number of interpolation points for the computation of the coefficients of a Newton two variable interpolating polynomial of a polynomial $f$ with the the following known characteristics:

$$
\begin{aligned}
& \text {-upper bound of the total degree } n=\operatorname{deg}[f(x, y)] \\
& \text {-upper bound of degree for the variable } x \text { (resp. } y), k_{1}= \\
& \left.\operatorname{deg}_{x}[f(x, y)] \text { (resp. } k_{2}=\operatorname{deg}_{y}[p(x, y)]\right)
\end{aligned}
$$

As far as we know, from the previous section, we have three choices for the selection of this set : a) triangular basis, b) rectangular basis and c) polygonal basis. Since the polygonal basis is coming from the intersection of the triangular and the rectangular basis, is always a subset of these two bases and therefore has the least interpolation points. As we shall see below, under certain conditions the polygonal base coincide with one of these two bases. 
Lemma 3.Let the Newton two-variable interpolating polynomial $p(x, y)$ has total degree $n$, upper bound degree for variable $x$ (resp. y), $k_{1}$ (resp. $k_{2}$ ). Then, it holds that

$$
n \leq k_{1}+k_{2} \leq 2 \cdot n
$$

Proof. The polynomial $p(x, y)$ has total degree $n$, when the maximum value of the summation of the exponents of the term $x^{n_{1}} y^{n_{2}}$ is equal to $n$, namely, $n_{1}+n_{2}=n$. Since the greatest power of $x$ (resp. $y$ ) in $p(x, y)$ is $k_{1}$ (resp. $k_{2}$ ) we have that

$$
\left.\begin{array}{l}
k_{1} \leq n \\
k_{2} \leq n
\end{array}\right\} \Longrightarrow k_{1}+k_{2} \leq 2 \cdot n
$$

and

$$
\left.\begin{array}{l}
k_{1} \geq n_{1} \\
k_{2} \geq n_{2}
\end{array}\right\} \Longrightarrow k_{1}+k_{2} \geq n_{1}+n_{2}=n
$$

From these inequalities we conclude that

$$
n \leq k_{1}+k_{2} \leq 2 \cdot n
$$

Based on the above we conclude that the selection of the appropriate base depends on the structure of the polynomial and more specifically, on the connection between $n, k_{1}$ and $k_{2}$. We distinguish three possible cases: If $k_{1}+k_{2}=n$ then we have

$N=\left(\begin{array}{c}n+2 \\ n\end{array}\right)=\frac{(n+1)(n+2)}{2} \stackrel{\left(k_{1}+k_{2}=n\right)}{=}$

$$
=\frac{k_{1}^{2}}{2}+k_{1} k_{2}+\frac{3 k_{1}}{2}+\frac{k_{2}^{2}}{2}+\frac{3 k_{2}}{2}+1
$$

$\tilde{N}=\left(k_{1}+1\right)\left(k_{2}+1\right)=k_{1}+k_{2}+k_{1} k_{2}+1$

$\hat{N}=\left(\begin{array}{c}n+2 \\ n\end{array}\right)-\left(S_{n-k_{1}}+S_{n-k_{2}}\right) \stackrel{\left(k_{1}+k_{2}=n\right)}{=} k_{1}+k_{2}+k_{1} k_{2}+1$

with a consequence

$$
N-\tilde{N}=\frac{k_{1}^{2}}{2}+\frac{k_{1}}{2}+\frac{k_{2}^{2}}{2}+\frac{k_{2}}{2}>0 \quad \text { and } \quad \hat{N}=\tilde{N}
$$

Thus, the optimal basis is the rectangular basis (Figure 2).

If $k_{1}+k_{2}=2 \cdot n$ then from Lemma 3 we have that $k_{1}=k_{2}=n$ and

$N=\left(\begin{array}{c}n+2 \\ n\end{array}\right)=\frac{(n+1)(n+2)}{2}$

$\tilde{N}=\left(k_{1}+1\right)\left(k_{2}+1\right) \stackrel{\left(k_{1}=k_{2}=n\right)}{=}(n+1)^{2}$

$\hat{N}=\left(\begin{array}{c}n+2 \\ n\end{array}\right)-\left(S_{n-k_{1}}+S_{n-k_{2}}\right) \stackrel{\left(k_{1}=k_{2}=n\right)}{=} \frac{(n+1)(n+2)}{2}$

with a consequence

$$
\tilde{N}-N=\frac{n^{2}}{2}+\frac{n}{2}>0 \quad \text { and } \quad \hat{N}=N
$$

thus, the optimal basis is the triangular basis (Figure 3).

Finally, if $n<k_{1}+k_{2}<2 \cdot n$ then the optimal basis is the polygonal basis (Figure 4 ).

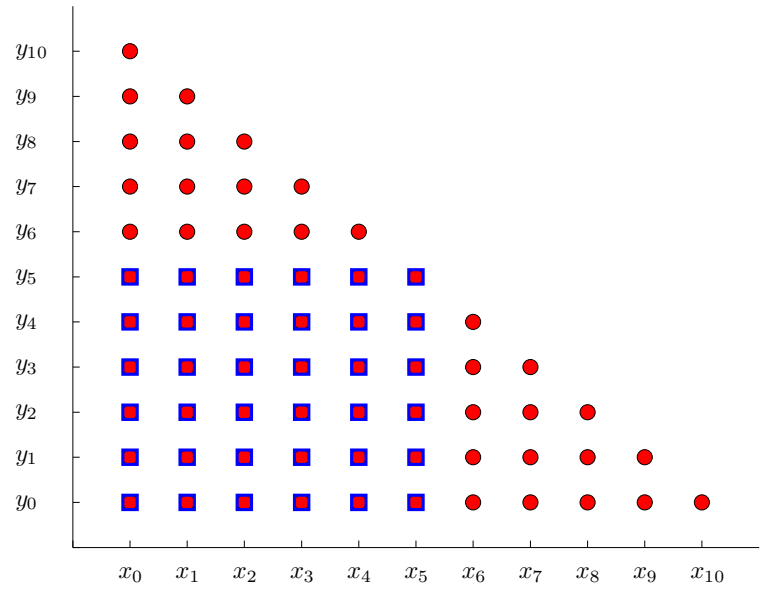

Fig. 2: The sets $S_{\Delta}^{(n)}$ (red circles) and $\tilde{S}_{\Delta}^{\left(k_{1}, k_{2}\right)}$ (blue squares) where $k_{1}+k_{2}=n$

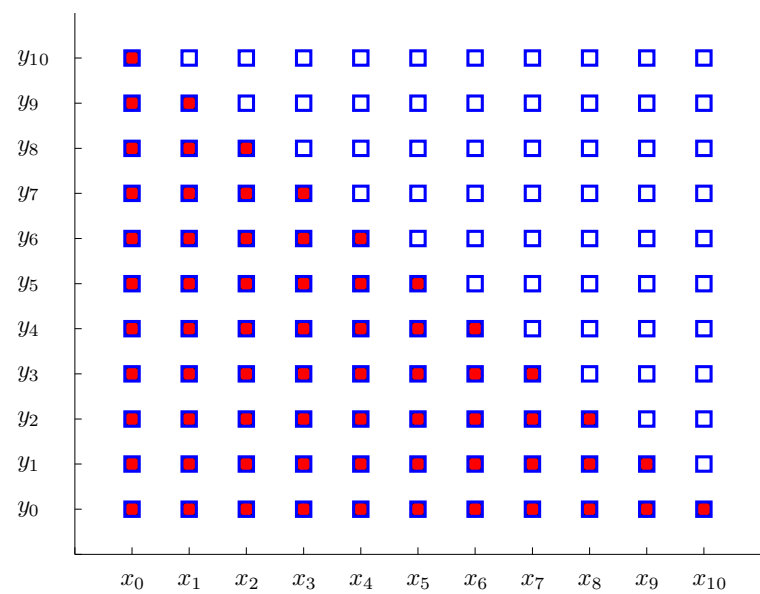

Fig. 3: The sets $S_{\Delta}^{(n)}$ (red circles) and $\tilde{S}_{\Delta}^{\left(k_{1}, k_{2}\right)}$ (blue squares) where $k_{1}+k_{2}=2 \cdot n$

Therefore, the optimal number of required interpolation points is given by the following formula

$N_{o}= \begin{cases}N=\left(\begin{array}{c}n+2 \\ n\end{array}\right) & \text { when } k_{1}+k_{2}=2 \cdot n \\ \tilde{N}=\left(k_{1}+1\right)\left(k_{2}+1\right) & \text { when } k_{1}+k_{2}=n \\ \hat{N}=\left(\begin{array}{c}n+2 \\ n\end{array}\right)-\left(S_{n-k_{1}}+S_{n-k_{2}}\right) & \text { when } n<k_{1}+k_{2}<2 \cdot n\end{cases}$

or equivalently as we already expected

$$
N_{o}=\hat{N}=\left(\begin{array}{c}
n+2 \\
n
\end{array}\right)-\left(S_{n-k_{1}}+S_{n-k_{2}}\right)
$$

where $n \leq k_{1}+k_{2} \leq 2 \cdot n$.

In the following two examples we present the application of the formula (7) and polygonal basis in problems of applied mathematics where the technique "evaluation-interpolation" is used. 


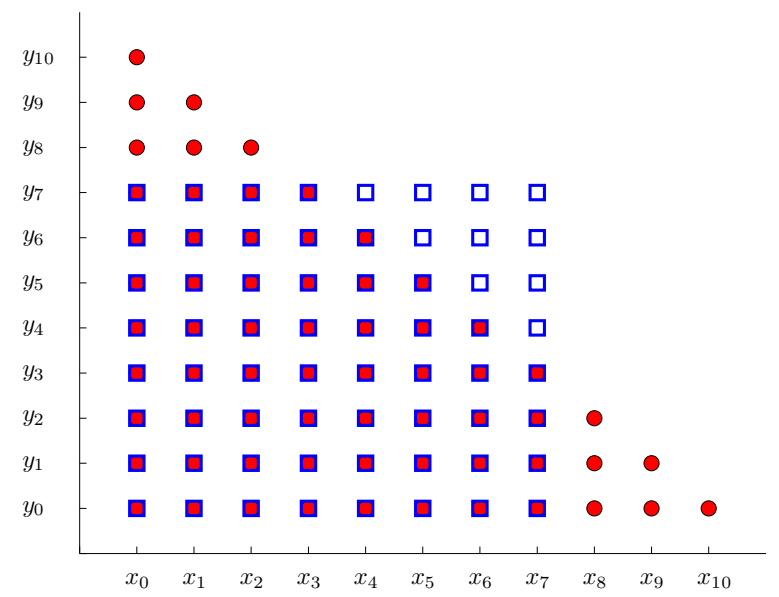

Fig. 4: The sets $S_{\Delta}^{(n)}$ (red circles), $\tilde{S}_{\Delta}^{\left(k_{1}, k_{2}\right)}$ (blue squares) and $\hat{S}_{\Delta}^{\left(n, k_{1}, k_{2}\right)}$ (red circles and blue squares) where $n<k_{1}+k_{2}<2 \cdot n$

Example 4(Computation of GCD). Let the two-variable polynomials

$$
f(x, y)=x^{9}-x^{4}-x^{5} y^{6}+y^{6}
$$

and

$$
g(x, y)=x^{11}-x^{6}-x^{5} y^{9}+y^{9}
$$

The total degree of the greatest common divisor $p(x, y)=\operatorname{gcd}\{f(x, y), g(x, y)\}$ has as an upper bound $n$ the greatest total degree in each polynomial i.e.

$$
n=\min \{\operatorname{deg}[f(x, y)], \operatorname{deg}[g(x, y)]\}=\min \{11,14\}=11
$$

Let $k_{1}$ (resp. $k_{2}$ ) be the upper bound of the degree of $x$ (resp. $y$ ) in $p(x, y)=\operatorname{gcd}\{f(x, y), g(x, y)\}$. Then

$$
k_{1}=\min \left\{\operatorname{deg}_{x}[f(x, y)], \operatorname{deg}_{x}[g(x, y)]\right\}=\min \{9,11\}=9
$$

and

$$
k_{2}=\min \left\{\operatorname{deg}_{y}[f(x, y)], \operatorname{deg}_{y}[g(x, y)]\right\}=\min \{6,9\}=6
$$

Therefore, we need to find a Newton two-variable interpolating polynomial with total degree $n=11$ and degree for variable $x$ (resp. $y$ ), $k_{1}=9$ (resp. $k_{2}=6$ ) with $n<k_{1}+k_{2}<2 \cdot n$. The least number of interpolation points that we need is

$$
N_{o}=\hat{N}=\left(\begin{array}{c}
n+2 \\
n
\end{array}\right)-\left(S_{n-k_{1}}+S_{n-k_{2}}\right)=60
$$

on polygonal basis instead to $N=78$ for the triangular basis and $\tilde{N}=70$ for the rectangular basis. An algorithm for the solution of this problem based on DFT interpolation techniques can be found in [14].

Example 5(Computation of the determinant). Consider the two-variable polynomial matrix $A(x, y)$ of the Example 1 with determinant $p(x, y)=\operatorname{det} A(x, y)$, having an upper bound of the total degree $n=27$ and upper bound $k_{1}=19$ (resp. $k_{2}=16$ ) of the degree of $x$ (resp. $y$ ). Since $n<k_{1}+k_{2}<2 \cdot n$, we can use a polygonal basis with

$$
N_{o}=\hat{N}=\left(\begin{array}{c}
n+2 \\
n
\end{array}\right)-\left(S_{n-k_{1}}+S_{n-k_{2}}\right)=304
$$

interpolation points instead of a triangular basis with $N=406$ interpolation points or a rectangular basis with $\tilde{N}=340$ interpolation points. The number of interpolation points is significantly important on the computation of the determinant of a two-variable matrix, since for its solution a respective number of computations of constant determinants is needed [13].

\section{Conclusions}

In certain types of Newton two-variable interpolation problems, we already have an estimation of the upper bound of the total degree $n$ of the interpolating polynomial $p(x, y)$ and the total degree of its variables $\left(k_{1}\right.$ (resp. $\left.k_{2}\right)$ in terms of $x$ (resp. $y$ )). In that cases, it was shown that it is less expensive in computational cost and memory, to use a basis of interpolation points that comes from the interconnection of the well known triangular and rectangular basis. This new polygonal base, use less interpolation points and under certain conditions between the degrees of the polynomial, coincides with either the triangular basis $\left(k_{1}+k_{2}=2 \cdot n\right)$ or the rectangular basis $\left(k_{1}+k_{2}=n\right)$. This new polygonal basis can be used in many problems of applied mathematics such as a) the greatest common divisor of two-variable polynomials and b) the determinant of a two-variable matrix e.t.c. by using the Newton interpolation algorithm proposed in [13]. Although, we study here the specific case where the function that we would like to approximate is already a polynomial, this polygonal basis can also be used for the approximation of any function if the pre-specified properties for the Newton bivariate interpolating polynomial are satisfied.

\section{Acknowledgement}

The authors wish to acknowledge financial support provided by the Research Committee of the Serres Institute of Education and Technology under grant SAT/IC/26062012-65/3.

\section{References}

[1] Laurene V. Fausett. Numerical Methods: Algorithms and Applications. Prentice Hall, 2002.

[2] J. Stoer, R. Bulirsch, R. Bartels, W. Gautschi, and C. Witzgall. Introduction to Numerical Analysis. Texts in Applied Mathematics. Springer, 2002.

[3] Biswa Datta. Numerical Methods for Linear Control Systems. Academic Press, 2003.

[4] G. E. Forsythe, M. A. Malcolm, and C. B. Moler. Computer Methods for Mathematical Computations. Prentice Hall, 1977.

[5] C. de Boor and A. Ron. On multivariate polynomial interpolation. Constructive Approximation, 6:287-302, 1990.

[6] M. Gasca and T. Sauer. On the history of multivariate polynomial interpolation, numerical analysis, 2000, vol. ii: Interpolation and extrapolation. Journal of Computational and Applied Mathematics, 122(1-2):23-35, 2000. 
[7] M. Gasca and T. Sauer. Polynomial interpolation in several variables. Advances in Computational Mathematics, 12:377-410, 2000.

[8] R. D. Neidinger. Multivariable interpolating polynomials in newton forms. In Joint Mathematics Meetings 2009, Washington D.C., January 5-8, 2009.

[9] P. J. Olver. On multivariate interpolation. Studies in Applied Mathematics, 116:201-240, 2006.

[10] G. M. Phillips. Interpolation and approximation by polynomials. Springer-Verlag, 2003.

[11] T. Sauer and Y. Xu. On multivariate lagrange interpolation. Mathematics of Computation, 64:1147-1170, 1995.

[12] M. Gasca and J. J. Martinez. Bivariate hermite-birkhof interpolation and vandermonde determinants. Numerical Algorithms, 2:193-199, 1992.

[13] Dimitris N. Varsamis and Nicholas P. Karampetakis. On the Newton bivariate polynomial interpolation with applications. Multidimensional Systems and Signal Processing, 2012.

[14] Panagiotis Tzekis, Nicholas Karampetakis, and Haralambos Terzidis. On the computation of the gcd of 2-d polynomials. International Journal of Applied Mathematics and Computer Sciences, 17(4):463-470, 2007.

[15] Nicholas Karampetakis and Alexandros Evripidou. On the computation of the inverse of a two-variable polynomial matrix by interpolation. Multidimensional Systems and Signal Processing, 23:97-118, 2012.

[16] A. Schuster and P. Hippe. Inversion of polynomial matrices by interpolation. IEEE Transactions on Automatic Control, 37:363-365, 1992.

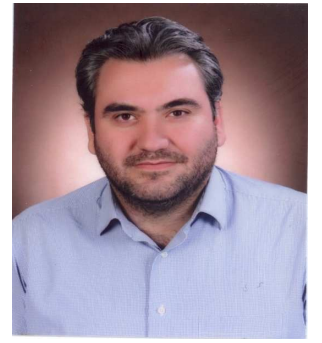

Dimitris N. Varsamis was born in Serres, Greece in 1976. He received the bachelor degree in Mathematics from the Department of Mathematics, University of Ioannina, Greece and the MSc in "Theoretical computer science, control and systems theory" from the Department of Mathematics, Aristotle University of Thessaloniki, Greece. He was awarded the Ph.D. at the Department of Mathematics (Aristotle University of Thessaloniki, Greece) in 2012, under the supervision of Associate Professor Nicholas Karampetakis. The subject of his doctoral thesis is "On the Development of Computational Methods for the solution of Control Theory problems". From 2001 to 2009 he was a scientific associate with Department of Informatics Communications, Technological Educational Institute of Serres, Greece. In October 2009 he was appointed as Lecturer in Department of Informatics Communications. Dr. Varsamis is the co-author of 20 articles in international journals and conference proceedings.His research interests include Scientific Programming, Computational methods, Control System Theory and Numerical Analysis.

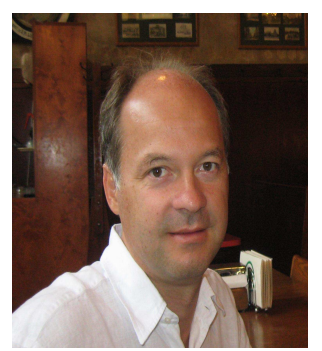

Nicholas P. Karampetakis was born in Drama, Greece in $1967 . \mathrm{He}$ received the bachelor degree in Mathematics and $\mathrm{Ph} . \mathrm{D}$. in Mathematics from the Department of Mathematics, Aristotle University of Thessaloniki, Greece in 1989 and 1993 respectively. From November 1994 to September 1995 he was a research associate with the Department of Mathematical Sciences, Loughborough University of Technology, England. From September 1995 to March 1999 he was a Research Associate with the Department of Mathematics, Aristotle University of Thessaloniki, Greece. From August 2000, to July 2009 he was an Assistant Professor to the same Department, whereas from July 2009, he has been Associate Professor. During the above periods he received many fellowships from the Greek Government, British Council and Engineering and Physical Sciences Research Council of England, and contributes in many research projects sponsored by the Greek Government and the European Union. His present research interests lie mainly in algebraic methods for computer aided design of control systems (CACSD), numerical and symbolic algorithms for CACSD, polynomial matrix theory and issues related to the mathematical systems theory. Dr. Karampetakis is a frequent contributor to the field in the form of journal articles, conference presentations and reviews. He is Senior Member of IEEE and a vice-Chair of the IEEE Action Group on Symbolic Methods for CACSD. He is also an Associate Editor a) of the Journal of Multidimensional Systems and Signal Processing and b) of the International Journal of Applied Mathematics and Computer Science. 


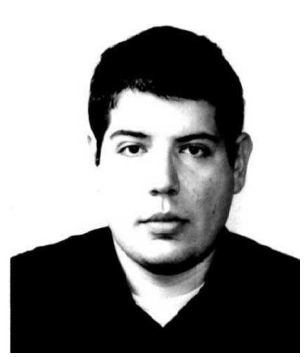

Paris Mastorocostas was born in Thessaloniki, Greece, in 1971. He received the Diploma and Ph.D. degrees in Electrical \& Computer Engineering from Aristotle University of Thessaloniki, Greece. Presently he serves as Professor at the Dept. of Informatics \& Communications, Technological Education Institute of Serres, Greece. His research interests include fuzzy and neural systems with applications to identification and classification processes, data mining and scientific programming. 